\title{
Role Of Magnetic Resonance Imaging With Diffusion Weighted Images In Assessment Of Patients with Pre-eclampsia In Pre And Postpartum Periods
}

\section{Tarek Mohamed M. Mansour ${ }^{1}$ MD; Sileem Ahmed Sileem ${ }^{2}$ MD; Mohamed M. El-Barody ${ }^{3}$ MD; Ahmed Okasha ${ }^{4}$ MD}

\section{* Corresponding Author: \\ Tarek Mohamed M. Mansour drtarekrad@gmail.com}

\section{Received for publication September 20, 2020; Accepted December 22, 2020; Published} online December 22, 2020.

\section{Copyright 2020 The Authors published by Al-Azhar University, Faculty of Medicine, Cairo, Egypt. All rights reserved. This an open-access article distributed under the legal terms, where it is permissible to download and share the work provided it is properly cited. The work cannot be changed in any way or used commercially.}

doi: $\underline{10.21608 / a i m j .2020 .43406 .1324}$ ${ }^{1}$ Radio-diagnosis department Faculty of Medicine, Al-Azhar University, Assuit, Egypt

${ }^{2}$ Obstetrics and Gynaecology department, Faculty of Medicine, AlAzhar University, Assuit, Egypt

${ }^{3}$ Radio-diagnosis department , South Egypt cancer institute, Assuit University, Assuit, Egypt.

${ }^{4}$ Radio-diagnosis department , South valley University, Qena, Egypt.

\begin{abstract}
BACKGROUND: We aimed to evaluate the predictive value of DWI in predicting the neurological manifestation of PE.

METHODS: We prospectively evaluated the radiological features of complications of pre-eclamptic patients presented from the Obstetrics department with peri-partum /eclampsia manifestations and confirmed by clinical examination and laboratory findings. Follow up was done within 10-14 days for patients with restriction of diffusion in DWI.

Results: The study included 44 patients with pre-eclampsia. The mean age of the included patients was $26.9 \pm 2.9$ years old. The admission systolic blood pressure was $181.8 \pm 15.2 \mathrm{mmHg}$. Twenty-nine patients $(65.9 \%)$ had neurological manifestations of pre-eclampsia at time of admission. Patients with neurological manifestations, revealed normal MRI findings in 7 patients $(24.1 \%)$ and abnormal features in 22 patients (75.9\%). In terms of atypical presentation of PE, restriction of diffusion accounted for $100 \%$ of patients with atypical PE. In addition, we found that the parietal lobe was the most frequently affected lobe, followed by the occipital lobe. The parieto-occipital regions were the most commonly affected areas and represented the typical site of PE. Comparing the gray and white matter involvement with the diffusion characteristics, it was noted that most cortical involvement revealed restricted diffusion of cytotoxic edema, all white matter involvements revealed enhanced diffusion of vasogenic edema, while mixed grey and white matter involvement revealed mixed diffusion properties (vasogenic and cytotoxic) with the cytotoxic edema located mainly in the grey matter $(\mathrm{p}<0.0005)$.
\end{abstract}

Conclusions: We suggest that DWI in PE patients with new neurological symptoms should be considered early in their management.

Key words: Pre-eclampsia; Neurological Manifestations; Diffusionweighted MRI.

Disclosure: The authors have no financial interest to declare in relation to the content of this article. The Article Processing Charge was paid for by the authors.

Authorship: All authors have a substantial contribution to the article.

\section{INTRODUCTION}

Preeclampsia (PE) is a common complication that affects around 5\% of pregnant women. It is typically present after 20 weeks of pregnancy with microvascular hemolysis, decreased platelet level, increased blood pressure, proteinuria, and impaired renal function. ${ }^{1,2}$

The exact PE etiology is still not well-known. Nevertheless, it was proposed that the disease is due to the ischemic placenta that arises from an abnormal remodeling of the spiral arteries between the maternal, and placental arteries. ${ }^{3,4}$
It is considered as one of the principal causes for maternal, fetal, and neonatal mortality with or without headache, dizziness, and nausea. ${ }^{5}$

Severe PE, which develops before 34 weeks of gestation, is characterized clinically as early-onset type. ${ }^{6}$

In $10 \%$ of women with PE, there are mental adverse effects, while in women with early-stage disease, this risk increases to $15 \%{ }^{7}$

If left untreated, pregnant women who have PE will be seriously affected by eclampsia, stroke, liver rupture, lung edema, or insufficient renal failure. ${ }^{8}$ 
Moreover, the risk of bronchopulmonary dysplasia and cerebral paralysis in children who come to ${ }_{10}$ mothers with PE is higher, caused by early delivery. ${ }^{9}$,

Neurological complications can also differ because of the differing pathogenesis between PE two types; however, previous studies have focused on neurological complications of patients suffering from $\mathrm{PE}$, but there is no research into comparing the neurological complications of early-onset patients against patients suffering from late-onset PE. ${ }^{11}$

Neuroimaging's diagnostic value for neurological diseases is self-evident; however, neuroimaging was criticized in pregnant patients because of the potential risk to fetus from computed tomography (CT). ${ }^{12}$ However, the modality has become widely applied in pregnant patients and has increased the detection rate greatly with continuous improvement in magnetic resonance imaging (MRI). ${ }^{13}$

However, the standard of selecting the imaging methods for various neurological manifestations is still not consistent. In addition, the clinical factors that influence imaging outcomes are still controversial. Recently, diffusion-weighted MRI (DW-MRI) introduced into clinical practice as a noninvasive method that can detect cerebral infarction hours before changes become apparent. ${ }^{14}$

Moreover, it can differentiate between cytotoxic edema form vasogenic edema. However, its predictive value in predicting the neurological complications of $\mathrm{PE}$ is not established yet. ${ }^{15}$ Therefore, in this study, we aim to evaluate the predictive value of DWI in predicting the neurological manifestation of PE.

\section{PATIENT AND MATERIALS}

The present manuscript was approved by local ethics committee and prepared in concordance with the recommendations of Strengthening the Reporting of Observational studies in Epidemiology (STROBE). ${ }^{16}$

\section{Study Design and Patients:}

A prospective study was conducted on 44 patients presented from the Obstetrics department with peripartum pre-eclampsia/eclampsia manifestations as confirmed by clinical examination and laboratory findings- and they were referred to the radiology department during the period from January 2019 to December 2019. Follow up was done within 10-14 days for patients with restriction of diffusion in DWI. We excluded patients with poor diagnostic quality images due to patient non- cooperation; patients with absolute contra-indications for MRI examination such as patients with metallic surgical clips, joint prothesis, metallic bone plates, or patients who have cardiac pacemakers or surgical clips in or around the eyeballs; and/or patients with history of claustrophobia.

\section{MRI Examination and Data Collection}

Eligible patients underwent detailed history taking regarding the onset and course of pre-eclampsia and of associated symptoms as well as signs. All patients were investigated for laboratory parameters such as renal function tests, platelet count, liver function tests, coagulation profile and fundoscopy. Then, all patients were examined on a $1.5 \mathrm{~T} \mathrm{GE}$ signa Excite machine (GE Healthcare, Waukisha, WI). The range of days between the first presentation and having the MRI study was 2-3days.

The examination included a customized brain MRI study composed of FLAIR, T1 weighted, T2 weighted images and axial DWI. Follow up MRI followed the same sequences. Gadolinium enhanced T1 weighted image and magnetic resonance venography (MRV) were done for patient with venous infarction for confirmation of the diagnosis (Table 1).

\begin{tabular}{|c|c|}
\hline MRI Sequence & Parameters \\
\hline FLAIR & $\begin{array}{l}\text { Axial plane, } \mathrm{TR} / \mathrm{TE} / \mathrm{TI} \text { : } \\
7575 / 110 / 1593 \text { msec, slice } \\
\text { thickness/inter-slice gap: } 8 / 8 \mathrm{~mm} \text {, } \\
\text { and matrix: } 224 \text { x } 160 .\end{array}$ \\
\hline T1WI & $\begin{array}{l}\text { Sagittal plane, TR/TE: } 560 / 15 \\
\text { msec, slice thickness/inter-slice } \\
\text { gap: } 6 / 7 \mathrm{~mm} \text {, and matrix: } 224 \mathrm{x} \\
\text { 192. }\end{array}$ \\
\hline DWI & $\begin{array}{l}\text { Axial plane, TR/TE: } 280 / 118 \\
\text { msec, slice thickness/inter-slice } \\
\text { gap: } 8 / 8 \mathrm{~mm}, \mathrm{~b} \text { value: } 750 \\
\text { s/mm2, and matrix: } 112 \times 96 \text { with } \\
\text { calculation of the ADC map. }\end{array}$ \\
\hline Gadolinium & $\begin{array}{l}\text { (Dimegluminegadopentate- } \\
\text { (Magnavist)) was given } \\
\text { intravenously in a dose of } 10 \mathrm{ml}\end{array}$ \\
\hline
\end{tabular}

Table 1: MRI Sequences Parameters

\section{Study's outcomes:}

The primary outcome in the present study was the MRI and DWI features of patients presented with neurological complications of pre-eclampsia.

\section{Statistical Analysis:}

SPSS version 19 (SPSS Inc., Cary, NC) was used for the statistical analysis. Descriptive analysis was used, first, including mean and standard deviation for quantitative data, e.g. age, systolic and diastolic blood pressure. Frequency tables were used for qualitative data, e.g. number of presenting symptoms and signs. Independent sample T-test was used to compare the difference of means in quantitative data, i.e. age, systolic and diastolic blood pressure. Chi square test was used for qualitative data. Spearman 
correlation test was used to test the correlation

\section{RESULTS}

The mean age of the included patients was $26.9 \pm 2.9$ years old. The admission systolic blood pressure and diastolic blood pressure were $181.8 \pm 15.2$ and $97.7 \pm$ $8.6 \mathrm{mmHg}$, respectively. Twenty-nine patients $(65.9 \%)$ had neurological manifestations of preeclampsia at time of admission. Fits was the most common presenting symptom. It was recorded in $86.7 \%$ of Patient. Altered conscious level was noted in $36.4 \%$ of patients. Visual disturbance (including blurring of vision) accounted for $40.9 \%$ of patients. Comparing the clinical data between patients with and patients without neurological manifestation, it was found that there was a significant difference between both groups regarding the mean systolic and diastolic blood pressures as well as the proteinuria $\mathrm{p}$ $<0.05$; (Table 2).

Patients with neurological manifestations, revealed normal MRI findings in 7 patients $(24.1 \%)$ and abnormal features in 22 patients (75.9\%).Patients with neurological manifestations who revealed abnormal MRI were classified according to the radiological findings in our study into patients with typical posterior reversible encephalopathy syndrome (PRES) accounting for $27.5 \%$, patients with atypical PRES accounting for $41.3 \%$, one patient with venous infarction accounting for $3.4 \%$ and also one patient with subarachnoid hemorrhage accounting for $3.4 \%$. All patients with atypical PRES had restriction of diffusion figure (1).

In addition, we found that the parietal lobe was the most frequently affected lobe, followed by the occipital lobe. The parieto-occipital regions were the most commonly affected areas and represented the typical site of PE table (3).

Patient's characteristics were presented in (Table-1). There was no statistically significant difference between both groups regarding age, sex, BMI, comorbidities and urologic surgical history, ( $p$-value $>0.05$ ) The stone characteristics are shown in table(2).

In this study, complete staghorn stones represented (24\%) in OSS group and (20\%) in PNL group while partial staghorn stones represented $(67 \%)$ in OSS group and $(80 \%)$ in PNL group with no significant difference.

In OSS group, the used technique was extended pelolithotomy in 20 cases, pyelolithotomy plus one or two nephrotomies in 4 cases and anatrophic between the occurrence of fits and blood pressure.

nephrolithotomy in 1 case. In PNL group, the access was achieved through one punctured calyx in 22 cases and through two calyces in 3 cases.

There is statistically significant difference between both groups regarding to the operative time with shorter duration for OSS group $(131.48 \mathrm{~min}+10.2)$ vs $(174.63 \mathrm{~min}+14.9)(\mathrm{p}$-value $<0.001)$.

Mean creatinine rise in OSS group was $(0.19 \pm 0.1$ $\mathrm{mg} / \mathrm{dl})$ and in PNL group was $(0.14 \pm 0.07 \mathrm{mg} / \mathrm{dl})$ which was statistically significant $(\mathrm{p}$-value $=0.04)$. Regarding mean operative $\mathrm{Hb}$ loss, it was $(1.5 \pm 0.85$ $\mathrm{mg} / \mathrm{dl})$ in OSS group versus $(1.65 \pm 0.90 \mathrm{mg} / \mathrm{dl})$ in PNL group with no significant difference table (7).

There is no statistically significant difference between study groups as regards the different complications, either intraoperative or postoperative and also according to modified Clavien postoperative grades of complications table (8).

Stone-clearance in OSS group was (92\%) and in PCNL group was (84\%) with no significant difference.

Hospital stay and recovery time were significantly shorter in PNL group than in OSS group.

Comparing the gray and white matter involvement with the diffusion characteristics, it was noted that most cortical involvement revealed restricted diffusion of cytotoxic edema, all white matter involvements revealed enhanced diffusion of vasogenic edema, while mixed grey and white matter involvement revealed mixed diffusion properties (vasogenic and cytotoxic) with the cytotoxic edema located mainly in the grey matter $(\mathrm{p}<0.0005)$ table (4).

The ADC values recorded from the regions of cytotoxic edema ranged from 0.12 to $7.55 \times 10-11$, with an average of $3.95 \times 10-11$.The ADC value recorded from the single venous infarction lesion was $6.98 \times 10-12$. All patients with DWI evidence of cytotoxic edema underwent follow up MRI studies after 2 weeks. The lesions were fully reversible except in one patient. The residual infarct in this patient was much smaller than the initial area of restricted diffusivity. The ADC value of the irreversible lesion was $6.2 \times 10-11$, while the mean ADC value of reversible lesions was $3.95 \times 10-11$. There was no significant difference between both numbers $(\mathrm{p}=0.627)$. 

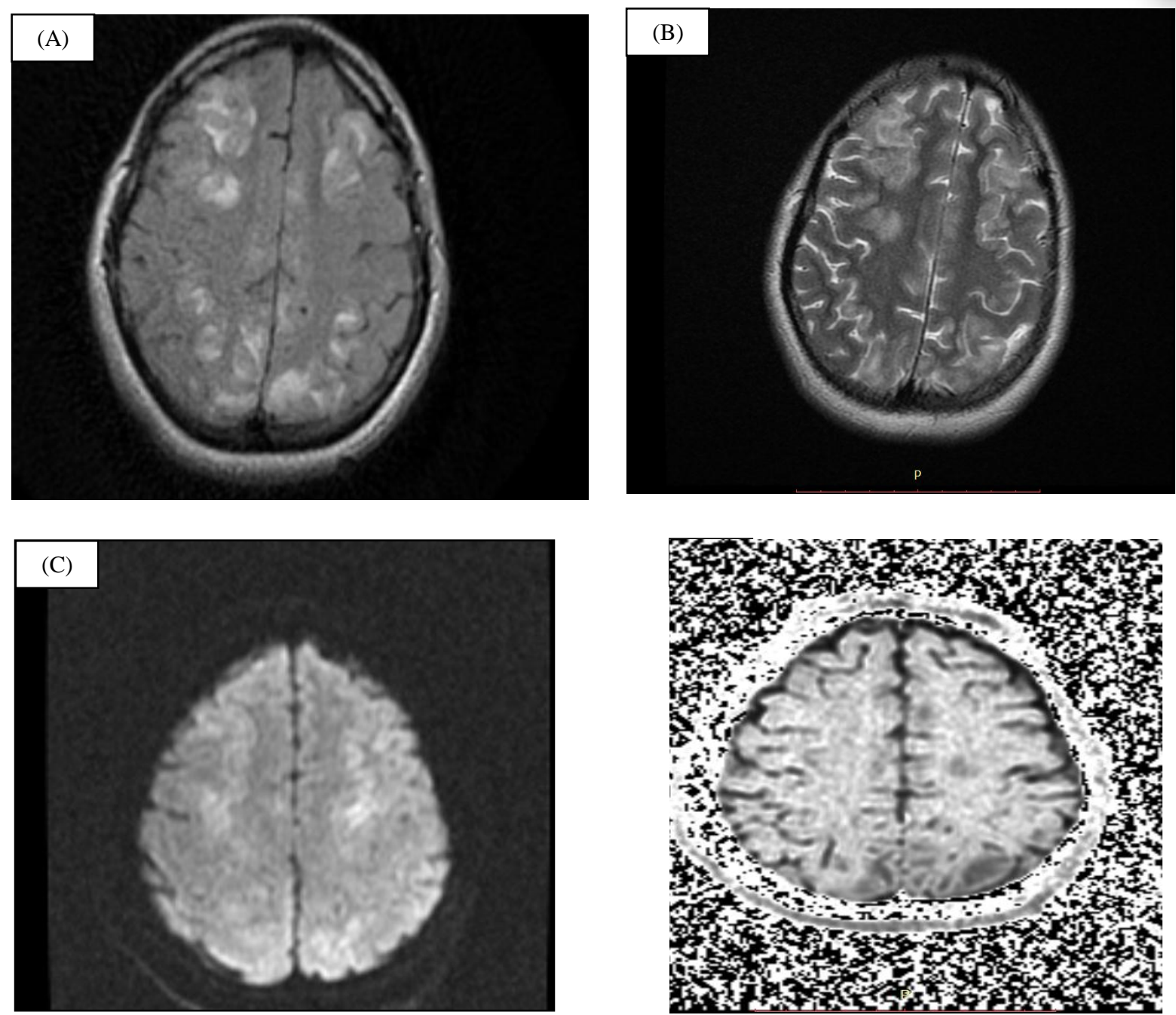

Figure 2: (a) Axial FLAIR shows abnormal high signal intensity area seen involving both parietal regions. (b) Axial T21WI shows abnormal high signal intensity areas in parietal region. (c) Axial DWI shows abnormal high signal intensity area seen involving the parietal region confirmed on the (d) ADC map which shows reduced ADC value. Interpretation: abnormal signal intensity areas are consistent with PRES which shows typical regional distribution (in parietal region). On DWI not all abnormal signal intensity areas seen in FLAIR images show restriction in diffusion denoting the presence of two types of edema (vasogenic and cytotoxic).

\begin{tabular}{|l|l|}
\begin{tabular}{|l} 
Number of patients $=44$ \\
Presenting symptoms
\end{tabular} & $\mathbf{N}(\boldsymbol{\%})$ \\
\hline Fits & $\mathbf{2 6}(\mathbf{5 9 . 1 \% )}$ \\
\hline Blurring of vision & $\mathbf{1 8}(\mathbf{4 0 . 9 \% )}$ \\
\hline Altered consciousness & $\mathbf{1 6}(\mathbf{3 6 . 4 \%})$ \\
\hline Hemiparesis & $\mathbf{3}(\mathbf{6 . 8 \%})$ \\
\hline Coma & $\mathbf{2}(\mathbf{4 . 5} \%)$ \\
\hline No neurological symptoms & $\mathbf{1 5}(\mathbf{3 4 . 1 \% )}$ \\
\hline
\end{tabular}

Table 2: The presenting symptoms in patients with pre-eclampsia (number and percent): 


\begin{tabular}{|c|c|}
\hline Regions affected & Number \\
\hline Parietal regions & $\mathbf{1 9}(\mathbf{9 5 \% )}$ \\
\hline Occipital regions & $\mathbf{1 8}(\mathbf{9 0 \%})$ \\
\hline Temporal regions & $\mathbf{1 0}(\mathbf{5 0 \% )}$ \\
\hline Frontal regions & $\mathbf{8}(\mathbf{4 0 \%})$ \\
\hline Basal ganglia & $\mathbf{4}(\mathbf{2 0 \%})$ \\
\hline Infra-tentorial & $\mathbf{2 ( 1 0 \% )}$ \\
\hline regions(cerebellum) & \\
\hline
\end{tabular}

Table 3: The distribution of signal abnormalities among the anatomical regions of the brain in relation to the number of the patients with PRES whether typical or atypical:

NB: Bilateral affection of the same anatomic region was considered as a single region involvement.

\begin{tabular}{|c|c|c|}
\hline & $\begin{array}{l}\text { Patients with PRES } \\
(\mathbf{N}=20)\end{array}$ & \\
\hline & $\mathbf{N}$ & $\%$ \\
\hline $\begin{array}{l}\text { Sites } \\
\text { - Mixed grey and white matter } \\
\text { - Sub-cortical white matter only } \\
\text { - Grey matter only }\end{array}$ & $\begin{array}{l}9 \\
7 \\
4\end{array}$ & $\begin{array}{l}45 \% \\
35 \% \\
20 \%\end{array}$ \\
\hline
\end{tabular}

Table 4: The distribution of grey and white matter involvement among patients with PRES $(n=20)$

\section{DISCUSSION}

It was known that healthy pregnant females undergo an elevation in the volume of the lateral ventricles at the moment of placental implantation, while the volume of both white and gray matter is reduced. ${ }^{17,18}$ A recent study showed that patients with PE had had smaller brain volumes and larger degrees of atrophy decades $^{19}$.

Moreover, patients with PE exhibit augmented cerebral perfusion pressure in the anterior, posterior, and middle cerebral arteries. ${ }^{20,21}$

which results in decreased cerebrovascular resistance and hyperperfusion, creating vasogenic edema and unfavorable hydrostatic pressure gradient. ${ }^{22,23}$

Regarding the white matter lesions, diagnostic imaging proposes that these lesions can persevere for years after the index pregnancy. ${ }^{24}$ These lesions distribute in the frontal lobes and tend to dominate in cases of early-onset PE. ${ }^{25}$

The presence of these lesions strongly associated with poor neurological outcomes in PE, especially when accompanied by intracranial hemorrhage, eclampsia, and cerebral edema. ${ }^{26}$

Technically, DWI is a very sensitive method to the diffusion properties of water molecules by applying a pair of pulsed magnetical field gradient to typical spin/echo and echo-planar imaging sequences. ${ }^{27}$ Water molecules that diffused an additional distance over the period between the paired pulses are less fully refocused by the second pulse and give a larger signal loss on DWI than slow-moving or limited water assemblies. ${ }^{28,29}$

Therefore, the limited diffusion areas on DWI appear relatively bright or hyperintense. ${ }^{30}$.

To the best of our knowledge, this was the first study in Egypt that assess the predictive value of DWI in predicting neurological complications of PE. Radiological screening showed that $66 \%$ of the patients were presented with neurological manifestations. Further, patients with neurological manifestations, revealed normal MRI findings in 7 patients $(24.1 \%)$ and abnormal features in 22 patients (75.9\%). The average of SBP and DBP was higher in patients with neurological manifestations $(p=0.001$ and $\mathrm{p}=0.002$, respectively). Moreover, they showed a higher incidence of proteinuria $(\mathrm{p}=0.001)$. 
In the study population, fits were the most common presenting symptoms followed by blurring of vision and altered conscious level. Only one case was reported with renal failure. In terms of atypical presentation of $\mathrm{PE}$, restriction of diffusion accounted for $100 \%$ of patients with atypical PE. In addition, we found that the parietal lobe was the most frequently affected lobe, followed by the occipital lobe. The parieto-occipital regions were the most commonly affected areas and represented the typical site of PE. The basal ganglia and the infra-tentorial regions were the least frequently encountered. Comparing the gray and white matter involvement with the diffusion characteristics, it was noted that most cortical involvement revealed restricted diffusion of cytotoxic edema, all white matter involvements revealed enhanced diffusion of vasogenic edema, while mixed grey and white matter involvement revealed mixed diffusion properties (vasogenic and cytotoxic) with the cytotoxic edema located mainly in the grey matter $(\mathrm{p}<0.0005)$. After two weeks of follow-up, we noticed that all lesions were fully reversible except in one patient. The residual infarct in this patient was much smaller than the initial area of restricted diffusivity figure (2).

Loureiro et al. ${ }^{15}$ enrolled 17 patients with severe PE to investigate whether DWI can predict the course of brain edema. They found that the sensitivity, specificity, and accuracy were (100\%), (75\%), and (94\%), respectively. Moreover, they showed an excellent agreement between the DWI and the follow-up MRI in the prediction of reversible brain lesions ( $\mathrm{k}$ value, 0.82 ).

Similar to our findings, they reported that all patients had lesions in the parieto-occipital lobes $(100 \%)$, followed by the frontal lobe (59\%). In terms of clinical presentation, all patients had vasogenic edema with microcirculatory vasodilatation, three patients had ischemic/cytotoxic edema with microvascular constriction, and two patients with cortical cytotoxic edema. After follow-up, 13 cases were reversible, and four cases were irreversible.

\section{CONCLUSION}

In conclusion, given the high mortality associated with intracranial events in PE and eclampsia patients, we suggest that neuroimaging in patients with severe PE developed with new neurological symptoms should be considered early in their management. DWI showed a significant role in distinguishing between potentially irreversible lesions that are linked with microvascular vasoconstriction from those lesions that are reversible on follow-up MRI.

\section{REFERENCES}

1. Gupte S, G. Wagh, Preeclampsia-Eclampsia, $J$. Obstet. Gynecol. India. 201) ; 64: 4-13. https://doi.org/10.1007/s13224-014-0502-y.

2. B.W.J. Mol, C.T. Roberts, S. Thangaratinam, L.A. Magee, C.J.M. De Groot, G.J. Hofmeyr, Pre- eclampsia, Lancet. 2016: 387: 999-1011. https://doi.org/10.1016/S0140-6736(15)00070-7.

3. B. Huppertz, Placental Origins of Preeclampsia, Hypertension. 2008 ; 51 : 970-975. https://doi.org/10.1161/HYPERTENSIONAHA.10 7.107607 .

4. X.L. Li, P.L. Guo, Y. Xue, W.L. Gou, M. Tong, Q. Chen, An analysis of the differences between early and late preeclampsia with severe hypertension, Pregnancy Hypertens. An Int. J. Women's Cardiovasc. Heal. 2016 ; 6: 47-52. https://doi.org/10.1016/j.preghy.2015.12.003.

5. X. Di, H. Mai, Z. Zheng, K. Guo, A.N. Morse, H. Liu, Neuroimaging findings in women who develop neurologic symptoms in severe preeclampsia with or without eclampsia, Hypertens. Res. 2018 ; 41 : 598-604. https://doi.org/10.1038/s41440-018-0051-3.

6. T. Tomimatsu, K. Mimura, M. Endo, K. Kumasawa, T. Kimura, Pathophysiology of preeclampsia: an angiogenic imbalance and longlasting systemic vascular dysfunction, Hypertens. Res. $2017 \quad ; \quad 40 \quad$ : $305-310$. https://doi.org/10.1038/hr.2016.152.

7. P. Von Dadelszen, B. Payne, J. Li, J.M. Ansermino, F.B. Pipkin, et al, Prediction of adverse maternal outcomes in pre-eclampsia: Development and validation of the fullPIERS model, Lancet. 2011 ; 377 : 219-227. https://doi.org/10.1016/S0140-6736(10)61351-7.

8. B. LaMarca, M. Cunningham, D. Cornelius, L. Amaral, Preeclampsia: long-term consequences for vascular health, Vasc. Health Risk Manag. 2015 ; 403. https://doi.org/10.2147/VHRM.S64798.

9. N. Principi, G.M. Di Pietro, S. Esposito, Bronchopulmonary dysplasia: clinical aspects and preventive and therapeutic strategies, J. Transl. Med. $2018 \quad$; $16 \quad 1636$. https://doi.org/10.1186/s12967-018-1417-7.

10. A. Menshawy, A. Ismail, M. Abdel-Maboud, A.A. El-din, A. Elgebaly, M. Gadelkarim, E.I. Bahbah, M.F. Abdelghany, A. Samy, A.M. Abbas, Effect of chlormadinone acetate versus drospirenone-containing oral contraceptives on the endocrinal features of women with polycystic ovary syndrome: Systematic review and metaanalysis of randomized clinical trials, J. Gynecol. Obstet. Hum. 2019. https://doi.org/10.1016/j.jogoh.2019.03.025.

11. S.K. Mishra, C.R.J.C. Newton, Diagnosis and management of the neurological complications of falciparum malaria, Nat. Rev. Neurol. 2009 ; 5: 189-198. https://doi.org/10.1038/nrneurol.2009.23.

12. T. Chansakul, G. Young, Neuroimaging in Pregnant Women, Semin. Neurol. 2017 ; 37 : 712 723. https://doi.org/10.1055/s-0037-1608939.

13. U.M. Reddy, R.A. Filly, J.A. Copel, Prenatal Imaging, Obstet. Gynecol. 2008 ; 112: 145-157. https://doi.org/10.1097/01.AOG.0000318871.9509 $0 . \mathrm{d} 9$. 
14. G.S. Chilla, C.H. Tan, C. Xu, C.L. Poh, Diffusion weighted magnetic resonance imaging and its recent trend-a survey., Quant. Imaging Med. Surg. 2015 ; 5 : 407-22. https://doi.org /10.3978/j.issn.2223-4292.2015.03.01.

15. R. Loureiro, C.C. Leite, S. Kahhale, S. Freire, B. Sousa, E.F. Cardoso, E.A. Alves, P. Borba, G.G. Cerri, M. Zugaib, Diffusion imaging may predict reversible brain lesions in eclampsia and severe preeclampsia: initial experience, Am. J. Obstet. Gynecol. 2003 ; 189 : 1350-1355. https://doi.org/10.1067/S0002-9378(03)00651-3.

16. E. Von Elm, D.G. Altman, M. Egger, S.J. Pocock, P.C. Gøtzsche, J.P. Vandenbroucke, S. Initiative, The Strengthening the Reporting of Observational Studies in Epidemiology ( STROBE ) Statement: Guidelines for reporting observational studies *, Int. J. Surg. 2014 ; 12: 1495-1499.

https://doi.org/10.1016/j.ijsu.2014.07.013.

17. K.M. Hillerer, V.R. Jacobs, T. Fischer, L. Aigner, The maternal brain: an organ with peripartal plasticity., Neural Plast. 2014 ; 574159. https://doi.org/10.1155/2014/574159.

18. A. Oatridge, A. Holdcroft, N. Saeed, J. V Hajnal, B.K. Puri, L. Fusi, G.M. Bydder, Change in brain size during and after pregnancy: study in healthy women and women with preeclampsia., AJNR. Am. J. Neuroradiol. 2002 ; 23: 19-26.

19. M.M. Mielke, N.M. Milic, T.L. Weissgerber, W.M. White, K. Kantarci, et al, Impaired Cognition and Brain Atrophy Decades After Hypertensive Pregnancy Disorders., Circ. Cardiovasc. Qual. Outcomes. 2016 ; 9: S70-6. https://doi.org/10.1161/CIRCOUTCOMES.115.00 2461.

20. K.P. Williams, S. Wilson, Variation in cerebral perfusion pressure with different hypertensive states in pregnancy., Am. J. Obstet. Gynecol. 1998 ; 179: 1200-3. https://doi.org/10.1016/s00029378(98)70131-0.

21. S. Riskin-Mashiah, M.A. Belfort, G.R. Saade, J.A. Herd, Cerebrovascular reactivity in normal pregnancy and preeclampsia., Obstet. Gynecol. 2001 ; 98: 827-32.

22. S. Riskin-Mashiah, M.A. Belfort, Preeclampsia is associated with global cerebral hemodynamic changes., J. Soc. Gynecol. Investig. 2005 ; 12: 253-6. https://doi.org/10.1016/j.jsgi.2005.01.002

23. M.J. Cipolla, Cerebrovascular function in pregnancy and eclampsia., Hypertens. (Dallas, Tex. 1979). 2007 ; 50: 14-24. https://doi.org/10.1161/HYPERTENSIONAHA.10 6.079442 .

24. M.J. Cipolla, N. Bishop, S.-L. Chan, Effect of pregnancy on autoregulation of cerebral blood flow in anterior versus posterior cerebrum. Hypertens. (Dallas, Tex. 1979). 2012 ; 60: 70511. https://doi.org/10.1161/HYPERTENSIONAHA.11 2.198952 .
25. P.G. Guyenet, The sympathetic control of blood pressure., Nat. Rev. Neurosci. 2006 ; 7: 335-46. https://doi.org/10.1038/nrn1902.

26. G.G. Zeeman, Neurologic complications of preeclampsia., Semin. Perinatol. 2009 ; 33: 166-72. https://doi.org/10.1053/j.semperi.2009.02.003.

27. K.J. van Everdingen, J. van der Grond, L.J. Kappelle, L.M.P. Ramos, W.P.T.M. Mali, Diffusion-Weighted Magnetic Resonance Imaging in Acute Stroke, Stroke. 1998 ; 29: 1783-1790. https://doi.org/10.1161/01.STR.29.9.1783.

28. D.G. Gregory, V.S. Pelak, J.L. Bennett, Diffusion-weighted magnetic resonance imaging and the evaluation of cortical blindness in preeclampsia, Surv. Ophthalmol. 2003 ; 48: 647650.

https://doi.org/10.1016/j.survophthal.2003.08.008.

29. X. Dong, J. Nao, Neurological manifestations and neuroimaging presentations in patients with severe preeclampsia: predisposing factors and clinical implications, Neurol. Sci. 2019;. https://doi.org/10.1007/s10072-019-03833-3.

30. M. Nelander, D. Hannsberger, I. SundströmPoromaa, L. Bergman, J. Weis, H. Åkerud, J. Wikström, A.K. Wikström, Assessment of cerebral perfusion and edema in preeclampsia with intravoxel incoherent motion MRI, Acta Obstet. Gynecol. Scand. 2018 ; 97 : 1212-1218. https://doi.org/10.1111/aogs.13383. 